\title{
Adaptive Spatial Query Processing Based on Uncertain Location Information ${ }^{\star}$
}

\author{
Yoshiharu Ishikawa ${ }^{1,2,3}$ \\ 1 Graduate School of Information Science, Nagoya University \\ 2 Information Technology Center, Nagoya University \\ Furo-cho, Chikusa-ku, Nagoya 464-8601, Japan \\ 3 National Institute of Informatics, Tokyo 101-0003, Japan \\ ishikawa@itc.nagoya-u.ac.jp
}

\begin{abstract}
In recent years, representation and management of uncertain data have gained much interests in the research field of database technologies. In this talk, we especially focus on spatio-temporal databases and consider the problems due to uncertain location information. Uncertainty of location information in spatio-temporal databases usually occur because of measurement errors, incorrect sensor readings, lack of signals, and movement of the objects, and results in non-accurate and non-reliable query results.

In this talk, we provide an overview of the current database technologies for managing uncertain location information. First, the background and the motivations are introduced. Some examples are taken from the fields of sensor databases and mobile applications. Second, a survey of interesting ideas in this field is provided. It covers not only uncertain location issues but also some related problems such as uncertain data streams and probabilistic frameworks for supporting uncertain queries.

Then we describe our past and current works for supporting adaptive spatial query processing considering uncertain location information. It includes a framework for probabilistic spatial queries, an indexing technique for uncertain spatial objects, and so on. We also show the application of the technologies to the decision support of mobile robots. Finally, the future research directions in uncertain location management are provided.
\end{abstract}

\footnotetext{
* This research was partly supported by the Funding Program for World-Leading Innovative R\&D on Science and Technology (First Program) and the Grant-in-Aid for Scientific Research (23650047) from JSPS.
} 